\title{
Current indications for magnetic resonance imaging
}

\author{
ABRAHAM PERA, DO \\ ATIS K. FREIMANIS, MD \\ JAMES B. MOORE, MD
}

Magnetic resonance is a wellestablished imaging modality for CNS, but it's utility for visualizing the rest of the body has not been appreciated fully or exploited yet. The authors believe that MRI will become one of the primary imaging modalities for the body during the next several years. Current and future indications for magnetic resonance imaging of the body are discussed.

Magnetic resonance imaging (MRI) of the body is in evolution. New techniques are being developed to overcome patient voluntary and involuntary (cardiac, respiratory, peristaltic) motion. Contrast agents that further improve the already excellent tissue differentiation and, thus, the sensitivity and specificity of diagnosis, are being developed. Cardiac and joint motion gated techniques provide the ability to visualize the heart or to watch a specific joint move through its range of motion, thereby allowing evaluation of function. As research continues and experience with the technique mounts, the ability of MRI to demonstrate normal and pathologic tissues better than any other present imaging modality appears certain.

In this paper, we focus on the various body systems for which MRI has been established as a useful imaging modality. In some areas it should be the primary choice, while in others it should be used to define an abnormality further. We also discuss the potential uses of MRI in upcoming years.

\section{Musculoskeletal system \\ Bone marrow}

Normal bone marrow primarily is composed of fat, which is imaged very well on magnetic resonance. When this fat is replaced by tumor or abnormal marrow, MRI demonstrates a different signal in the area. The sensitivity of this procedure is excellent. ${ }^{1}$ For example, in avascular necrosis of the femoral head, differentiation of abnormal from adjacent normal bone marrow is outstanding. This procedure is more sensitive than plain film roentgenography, computed tomography (CT), or radionuclide studies. $^{2-4}$ MRI should be the primary modality for evaluation of the various types of avascular necrosis of the hips, including Legg-Calve-Perthes disease (Fig 1).

Other conditions for which bone marrow signals are useful are lesions that may be secondary to ischemic necrosis, trauma, or hematologic problems, such as sickle cell disease. ${ }^{5}$ Although MRI

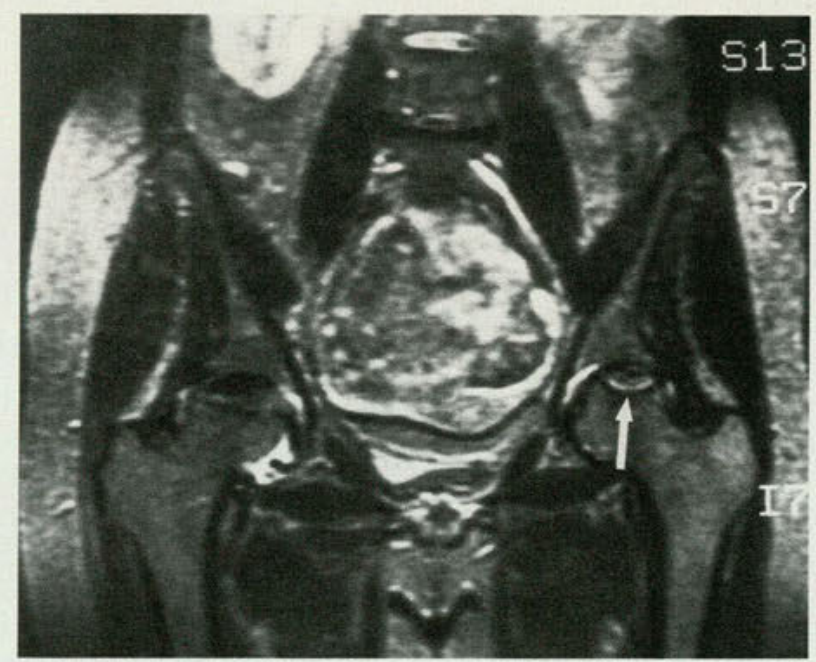

Fig 1. Old, right-sided avascular necrosis in a 22-year-old man. There is distortion of the weight-bearing portion of the femoral head. On the right, there is an elliptic rim of high signal (arrow) about the upper aspect of the femoral head. Plain films demonstrated a distorted right femoral head, but the left femoral head was shown to be completely normal. 


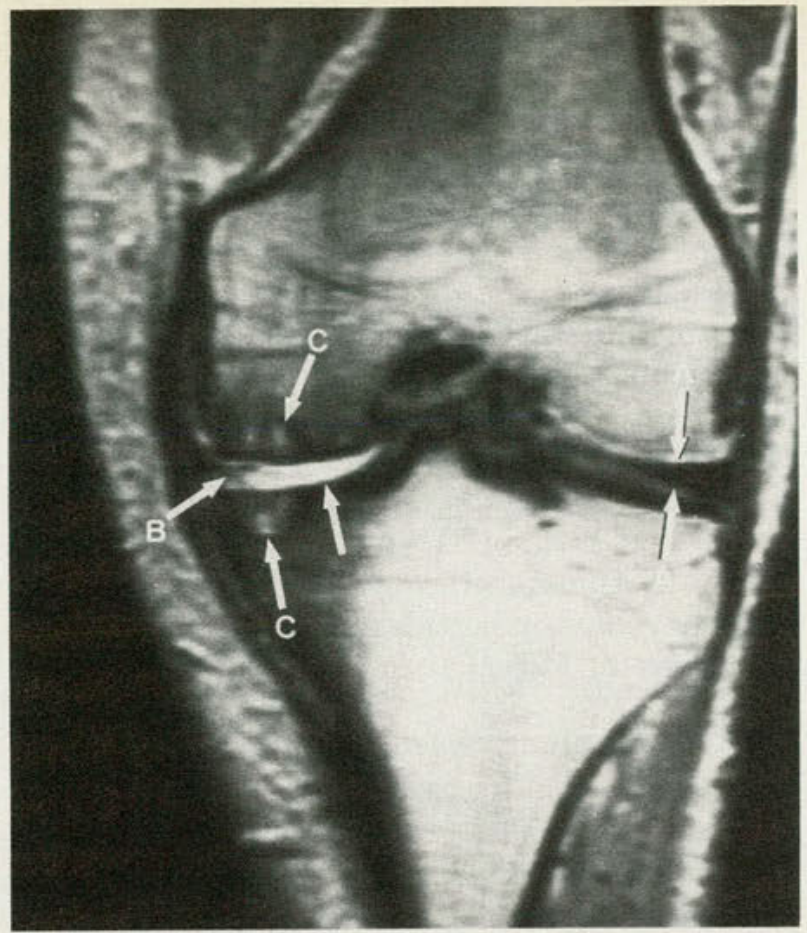

Fig 2. Knee of a 61-year-old man with degenerative joint disease. The plain arrow points to the medial compartment. A arrows show the relatively normal lateral meniscus. The medial meniscus (arrow B) has been destroyed completely. C arrows point to subchondral cysts.

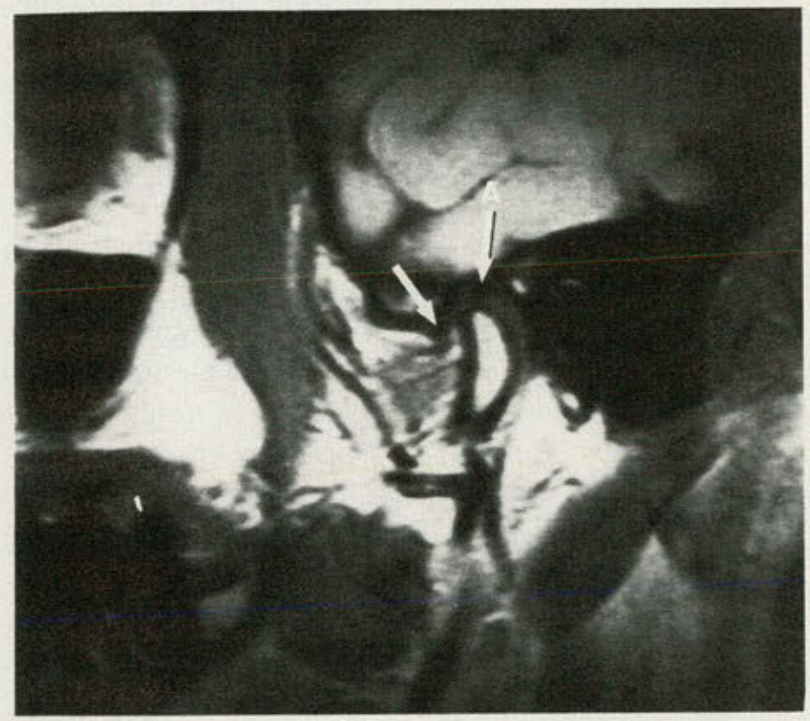

Fig 3. Normal TMJ, with the anterior (plain arrow) and posterior (arrow A) limbs of the disk in normal location in a closed mouth.

is not a tool for general screening for metastases to bone, if nuclear bone scanning identifies an area of suspicion and if the plain films are inconclusive, or if there is obscure, persistent bone pain, MRI may be used to investigate such a lesion further.

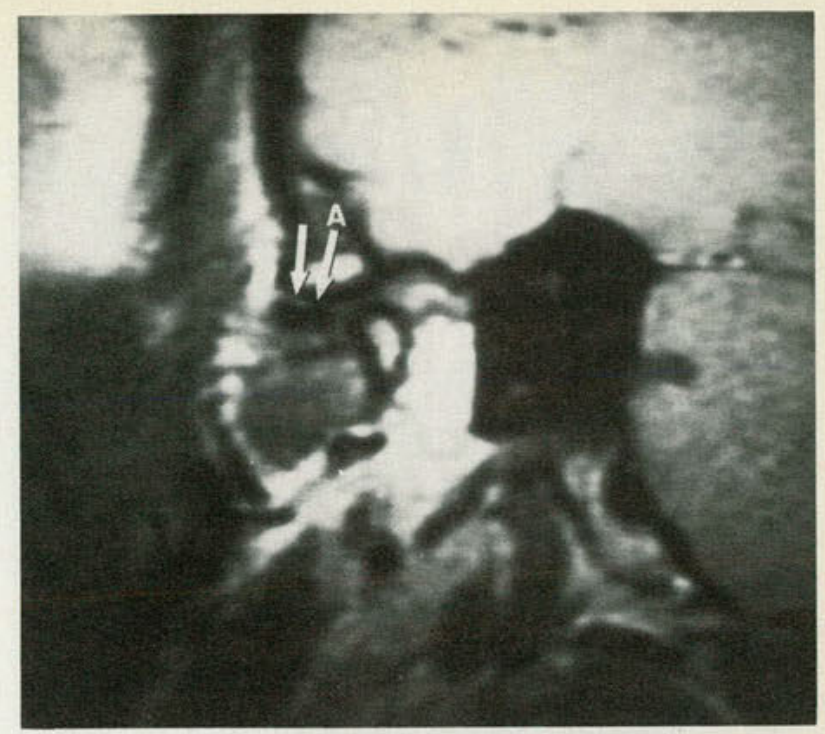

Fig 4. Anterior dislocation of the anterior (plain arrow) and posterior (arrow B) limbs of the meniscus, with the mouth in an open position.

Bone marrow infections are well demonstrated because of altered tissue characteristics of the involved areas. Edema produces a different magnetic resonance signal intensity than normal bone marrow with most pulse sequences, and it is differentiated easily from noninflamed tissue.

\section{Joints.}

The radiographic evaluation of joints is undergoing much change. Infective processes, effusions, and intra-articular masses are well demonstrated by MRI. Meniscal tears in the knee are demonstrated as sensitively as by arthroscopy, and ligamentous injuries are well defined. ${ }^{6,7}$ Constrast-injection arthrography of this joint should be replaced by MRI whenever possible (Fig 2).

Temporomandibular joint (TMJ) syndrome, which may account for local pain, cephalalgia, and chewing and speech difficulties, has been difficult to analyze radiugraphically. Imaging has been performed by plain film, linear tomography, arthrography, and CT with and without intra-articular contrast injections. As MRI becomes available, other modalities for evaluating internal derangement of the TMJ should be replaced. MRI enables detailed analysis of the disk and its motion relative to the head of the mandible and the mandibular fossa. In addition, joint effusion can be identified, and the integrity of surrounding ligamentous and muscular attachments can be evaluated. ${ }^{8,9}$ (Figs 3,4).

The shoulder, ${ }^{10}$ wrist $^{11}$, and ankle ${ }^{12}$ also are visualized well on MRI. In demonstration of rotator cuff 


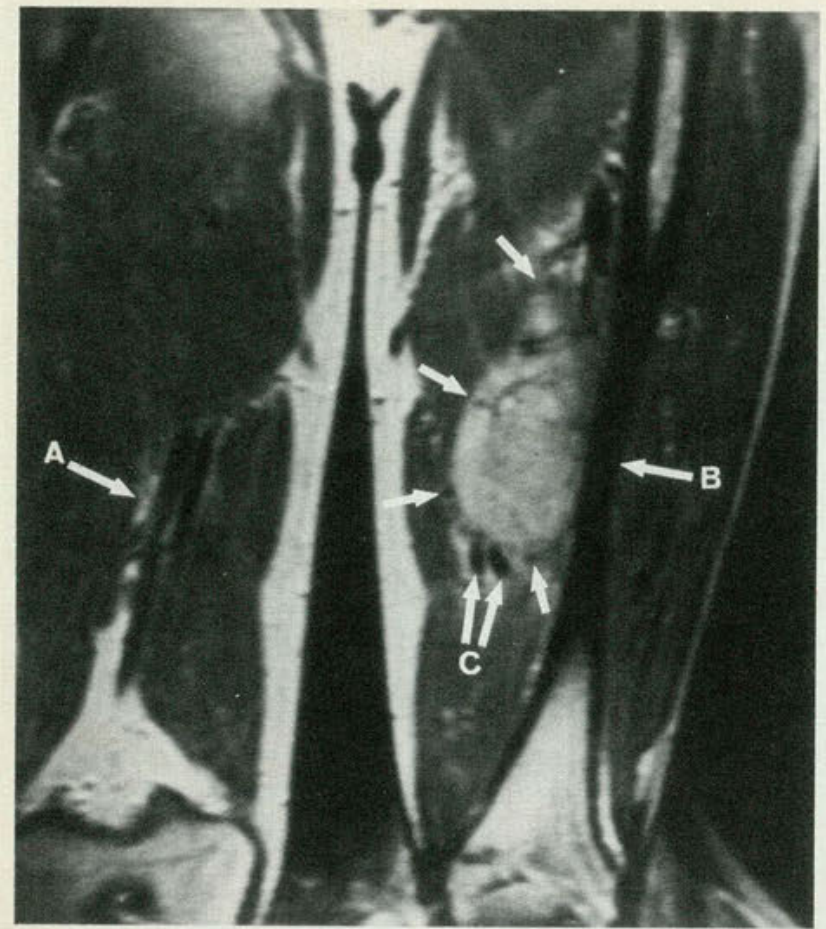

Fig 5. Five-centimeter malignant, fibrous histiocytoma in left medial thigh (plain arrows). The cortex of the femur (arrow B) is not disrupted. The vessels ( $\mathrm{C}$ arrows) were pushed by the mass but not invaded. A arrows demonstrate the normal vascular bundle of the right thigh.

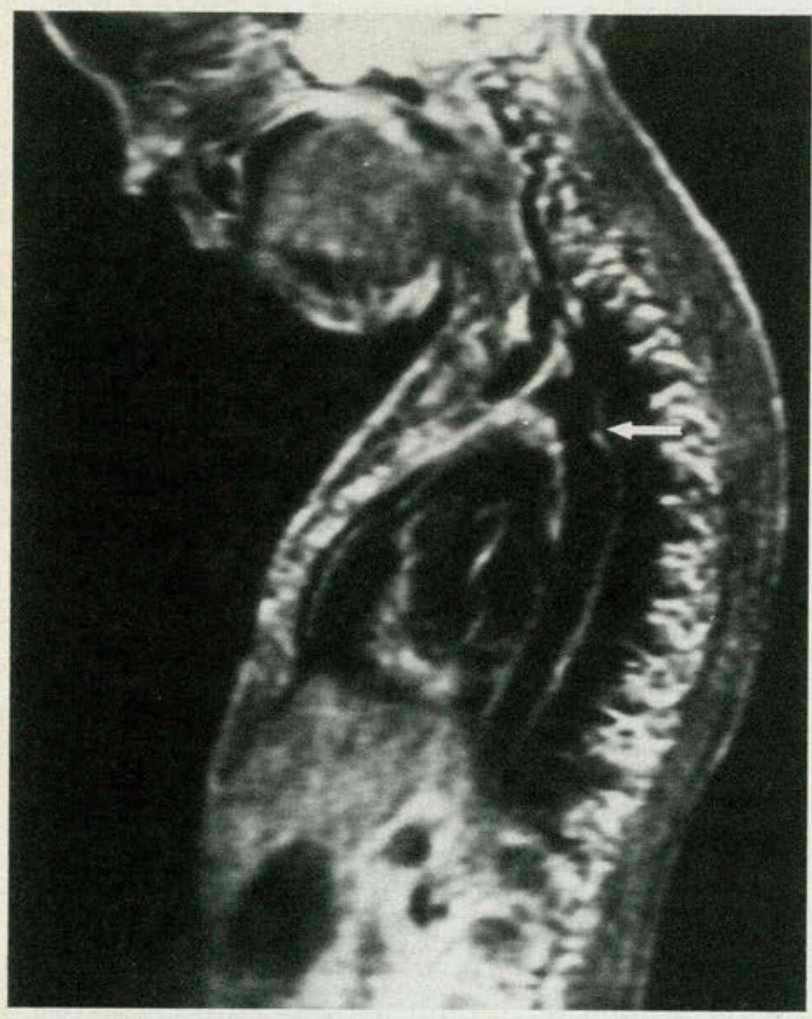

Fig 6. Previous balloon dilatation of a coarctation of the aorta in an 8-year-old child. Arrow points to remaining coarctation. tears in the shoulder, MRI may replace arthrography. ${ }^{10}$

Musculoskeletal tumors can be evaluated in three planes. The total extent of the mass and any intramedullary invasion of bone can be discerned. However, because magnetic resonance is relatively insensitive to bone, cortical bone destruction and intratumoral calcification are demonstrated poorly as compared with CT or roentgenography. But MRI does permit characterization of the components of the tumor (fat, necrosis, soft tissue) and their relationship to surrounding structures (vessels, nerves, joints) better than CT or plain roentgenography (Fig 5). The most efficacious site for biopsy and subsequent radiation portals for therapy can be identified best by MRI. ${ }^{12}$

\section{Cardiovascular system}

Although MRI only recently has joined the family of cardiovascular tests, it is rapidly coming to the forefront as a major imaging modality. The main reasons are its ability to electrocardiographically gate acquisitions of information (as in nuclear cardiology) and its ability to evaluate structures in motion by projecting the images in a sequential manner at a fast frame rate (cine mode). In addition, the ability to differentiate flowing blood from muscle without the need for injection of contrast agents allows imaging of the cardiac chambers. The inherently excellent tissue characterization allows imaging of ischemic areas of myocardium, and MRI's capability for volume measurements allows functional evaluation.

Spatial resolution of MRI is much superior to that obtained by isotope methods. In the future, magnetic resonance most likely will replace most other imaging modalities for cardiovascular investigation. ${ }^{14,15}$ Research in this area is now very active, and new applications are reported with the arrival of each new journal issue.

The heart chambers, pulmonary arteries, aorta, and even the valves can be demonstrated with excellent resolution. Myocardial wall thickening and thinning can be seen, as can pericardial effusions and masses. Aneurysmal defects and dissections of the heart chambers and great vessels can be imaged (Figs 6,7). In fact, in dissections, imaging by magnetic resonance is more accurate than angiography. The dissected portion of the lumen may contain a clot, which may not be demonstrated angiographically because of the lack of blood flow in the area. The clot and extraluminal extent of the lesion is demonstrated by MRI. The ability to perform volumetric measurement during the different parts of the cardiac cycle permits functional 


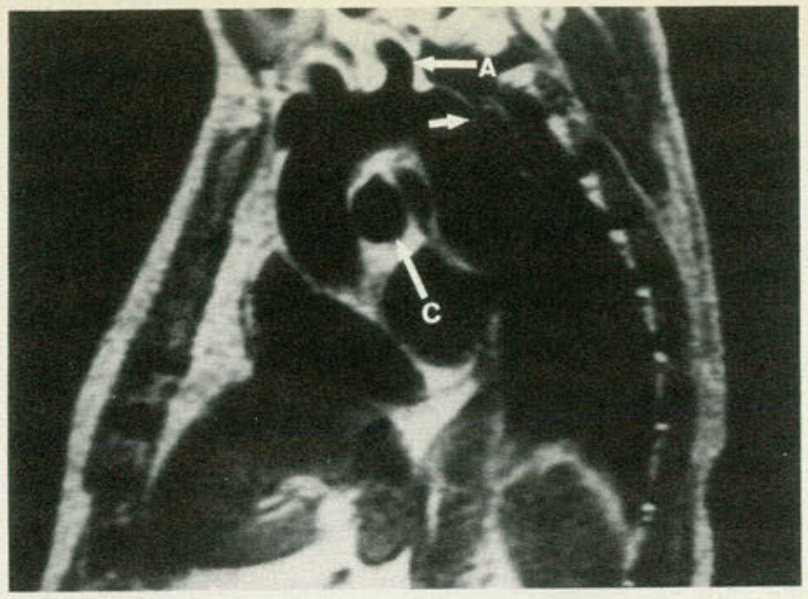

Fig 7. Traumatic dissection of the aorta immediately beyond the aortic arch in a 42-year-old man. Arrow A points to the left carotid artery, and the plain arrow points to a flap of aneurysm superiorly between the true and false channels (plain arrow). The left pulmonary artery also is demonstrated (arrow C).

evaluations such as stroke volume and the calculations of cardiac output and regurgitant flow. ${ }^{14}$

Myocardial infarction can be identified because of the increased signal in the ischemic areas on certain imaging pulse sequences. One recent hospital study ${ }^{15}$ demonstrated 23 of 25 consecutive myocardial infarctions. The proximal main coronary arteries frequently can be seen with present techniques. In the future, MRI may be indicated for evaluation of these vessels both before and after surgery. ${ }^{16}$ Three-dimensional displays of vascular structures by MRI that presently are being generated in preliminary research projects are comparable to most digital subtraction studies. This procedure does not require the injection of a contrast medium injection or other invasive procedure. Once it is generally available, the procedure most likely will be the screening modality of choice for evaluation of the major blood vessels of the neck and extremities. ${ }^{17}$

\section{Chest}

For evaluating the chest (excluding the heart), plain roentgenography and CT are excellent modalities. However, it is not possible to differentiate vessels from soft-tissue masses on plain films, and bolus intravenous contrast agent injection and fast (dynamic) scanning often are necessary for CT studies. MRI in the mediastinum and the hilar areas is as sensitive as CT. In addition, MRI does not require any intravenous contrast material, and it very accurately differentiates flowing blood in a vascular structure from soft-tissue masses.

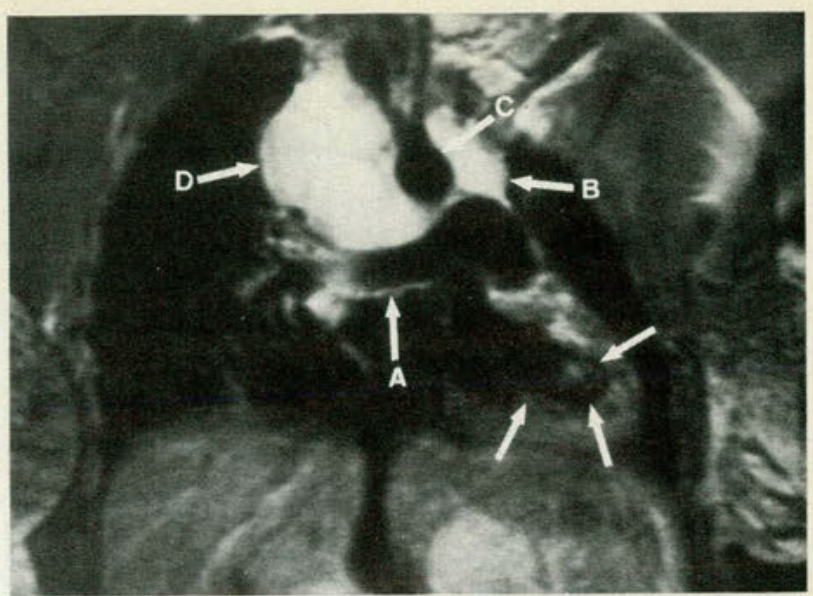

Fig 8. Lymphoma in a 24-year-old woman. Multiple lobular mediastinal lymph nodes surround the great vessels. These are of higher signal than adjacent fat, and, most likely, they represent active disease (arrows B, D,). The left ventricle (plain arrows), right pulmonary artery (arrow A), and coronal cut through the arch of the aorta (arrow $\mathrm{C}$ ) also are demonstrated.

The anatomic features also may be imaged in three planes, which provides more accurate localization. MRI's tissue characterization abilities, when combined with the potential for spectroscopy, eventually may allow differentiation of benign from malignant masses. ${ }^{18}$ This is especially important for the evaluation of patients with mediastinal or hilar lymphoma masses who have had previous therapy (Fig 8). For evaluation of nodules within the lung parenchyma, CT still is preferred to MRI.

\section{Abdomen \\ Gastrointestinal system}

The intestinal tract is not evaluated well by MRI or CT. The main reasons are peristaltic motion and nonconsistent distention of bowel. The advantages of $\mathrm{CT}$ are that extremely fast scanning can be performed and good intraluminal contrast agents are available to minimize these problems. MRI counterparts are not adequately developed at present. The evaluation of intraluminal contrast agents has just begun, and work is under way to develop similar short acquisition time scans.

Presently, small intraperitoneal pathologic fluid collections and masses are demonstrated more accurately by CT because of its better spatial resolution and faster scanning (with patients holding their breath during actual scanning). However, advantages of MRI, such as good differentiation of vascular structures and the ability to obtain images in three planes, will make it competitive once contrast agents and faster imaging acquisition methods are developed. 

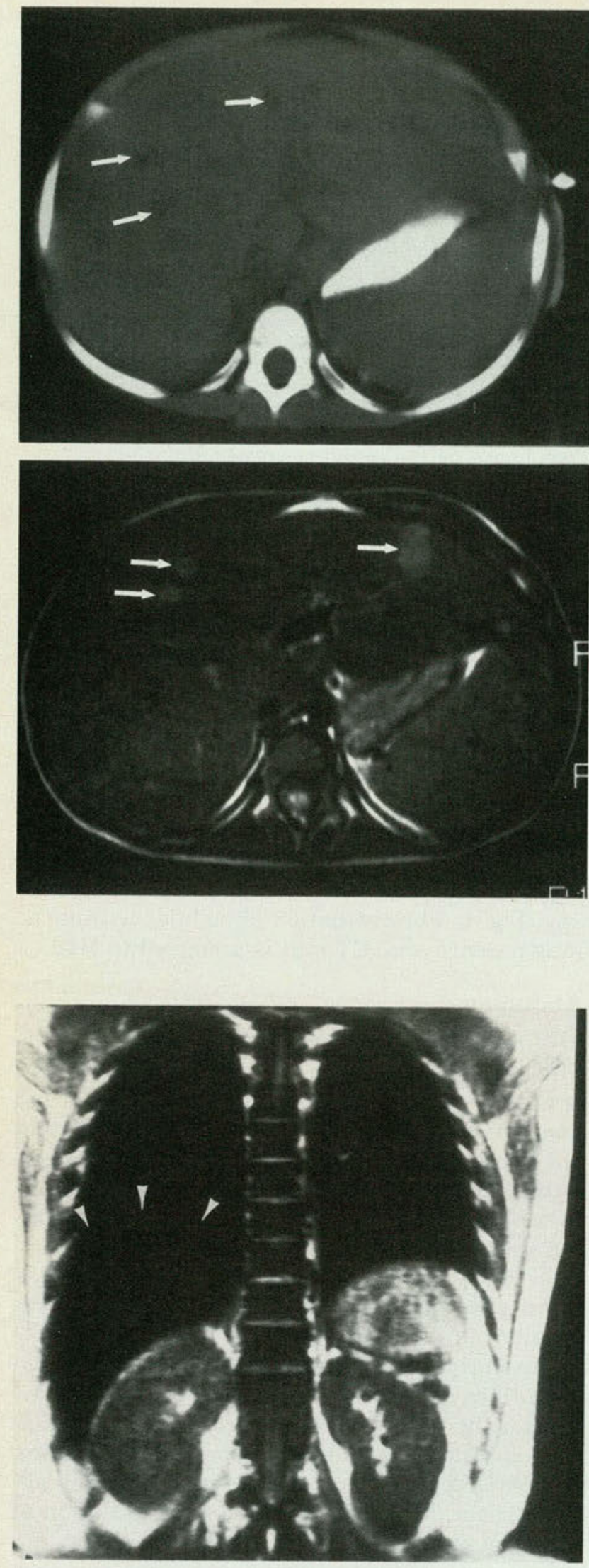

Fig 11. Sickle cell anemia and hemosiderosis in a 22-year-old black woman who had a history of multiple blood transfusions. The liver is extremely black (arrows) compared with a normal liver (Fig 8).
Figs 9, 10. CT scan (top) and MRI (bottom) of a 2-year-old girl with leukemia. The CT scan is without contrast because of the elevated creatinine level in this child. No abnormality was seen. The darker areas (arrows) in the liver are vessels. On MRI, three distinct areas of high signal (arrows) are consistent with focal fungal abscesses. The child had Candida albicans lesions of the skin. The liver lesions disappeared on a subsequent scan.

\section{Hepatobiliary system}

In the liver, masses are demonstrated well on MRI, and overall sensitivity to lesions may be better than with $\mathrm{CT}^{19}$ (Figs 9,10). Hemangiomas, which are very common incidental findings in the evaluation of the liver, have a characteristic signal on MRI and can be differentiated from other lesions, such as malignant tumors. This diagnosis is made with some difficulty by CT, ultrasonography, and arteriography..$^{20}$ Diffuse iron deposition in the liver (hemosiderosis) has a markedly low signal on MRI. This finding is striking and specific, and it can be used to quantify the amount of iron deposition in the liver ${ }^{21}$ (Fig 11).

\section{Spleen}

The spleen is demonstrated well on MRI. Focal lesions may be delineated. Diffuse involvement by lymphoma, which often is difficult to demonstrate radiographically by CT and ultrasonography, may be seen better with MRI. ${ }^{22}$

\section{Pancreas}

The normal pancreas is seen with some difficulty on MRI because of the similarity of its signal intensity to that of the surrounding fat and bowel. CT presently is the imaging modality of choice when pancreatic disease is suspected. With new developments, however, this area will become more amenable to imaging by magnetic resonance.

\section{Adrenal glands}

The adrenal glands can be seen with MRI. Masses can be identified as accurately as they are by CT. Some recent work has characterized adrenal lesions according to their signal intensity. There is a possibility that adrenal masses that are secondary to benign adenoma, metastasis, and pheochromocytoma may be differentiated by MRI signal intensity alone. ${ }^{23,24}$

\section{Renal system}

Many imaging modalities currently are available to evaluate the urinary system and to demonstrate the specific abnormalities suspected. MRI provides good visualization of the kidneys. The normal re- 


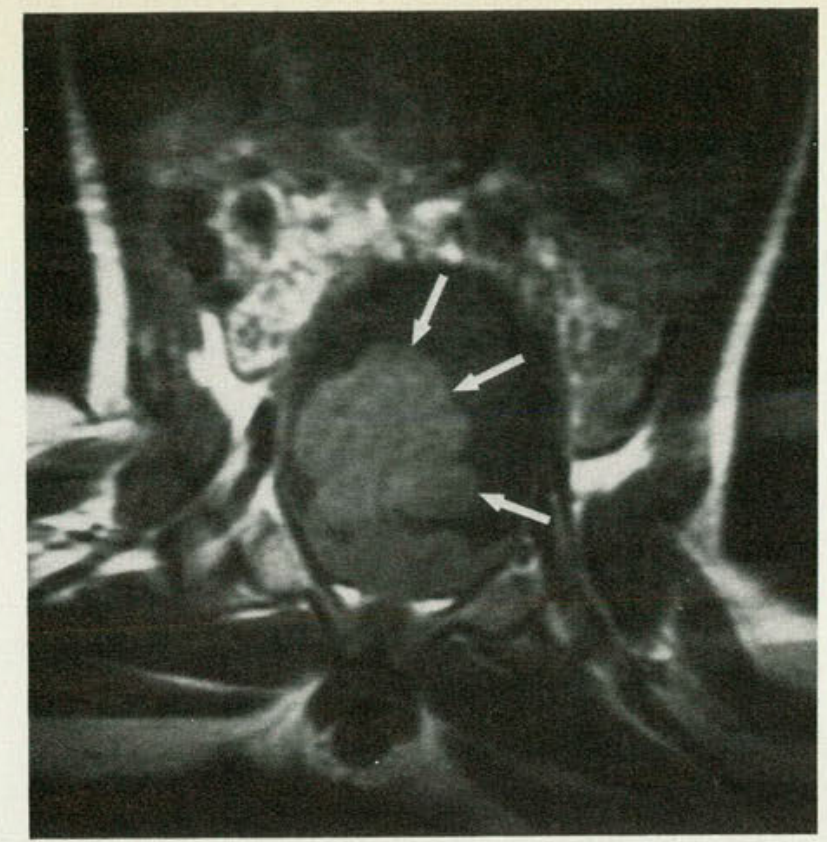

Fig 12. Sarcoma botryoides in an 18-month-old boy. A multilobular mass extends into the bladder lumen from the prostate gland and in a "grape cluster" configuration (arrows). After several courses of chemotherapy, the mass disappeared almost completely.

nal cortex shows a higher signal than the medullary portion, and, therefore, the two are differentiated easily. This difference in signal becomes poor with dehydration, renal transplant rejection, and renal artery thrombosis.

Conversely, with acute tubular necrosis (ATN), the medulla shows a higher signal than the cortex. This configuration would serve to separate ATN from a rejection in transplanted kidneys, which often is a clinical differential problem.

Paroxysmal nocturnal hemoglobinuria has been shown to decrease markedly the signal intensity in the cortex relative to that of the medulla. This disease, which is caused by complement-mediated red blood cell lysis, causes hemosiderin deposition in the cortex. Similarly to the liver hemosiderosis demonstrated in Fig 11, the resulting decreased signal in certain acquisitions makes it easily recognizable. ${ }^{25}$

MRI provides excellent visualization of vascular structures, and staging of renal cell carcinoma with extension of mass or clot into the inferior vena cava can be demonstrated accurately without the need for IV contrast injection. The disadvantage of MRI in visualizing the renal system is that compared with CT, it is relatively insensitive to calcifications.

\section{Pelvis}

Motion artifacts, which are the major cause of the degradation of images obtained by MRI in the ab-

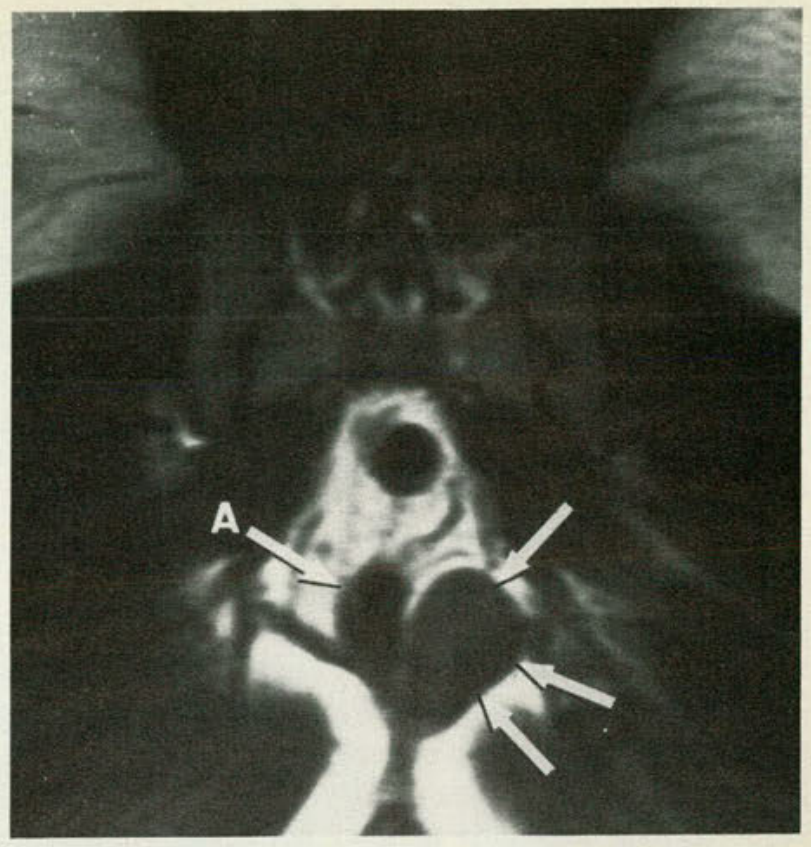

Fig 13. Left-sided pararectal mass (plain arrows) in a 33-yearold man. The lesion has a smooth-walled, benign cystic appearance, and it is histologically classified as a cystic teratoma. The normal rectum is pointed out by arrow A.

domen, are not as troublesome in the pelvis. Therefore, excellent views can be obtained in this area. The tissue contrast and resolution of fascial planes and the ability to differentiate vessels from solid mass give MRI the capability to perform well in staging of tumors in this region. In the bladder, when imaged in distention, the wall can be seen separate from the urine. Tumors and bladder wall have different signal characteristics; therefore, bladder invasion and extravesical spread of malignancy may be identified. The lack of bone artifacts in MRI makes it more advantageous than $\mathrm{CT}$ in this location (Fig 12).

For pelvic tumors, postoperative changes would be evaluated better with MRI than with CT because of MRI's ability to differentiate postoperative scarring from active tumor. Although screening for rectal tumors is performed best by other means, CT or MRI is best for staging of extension of disease (Fig 13).

The prostate gland can be imaged in three planes by MRI. Initial work to differentiate malignant disease from benign hyperplasia and inflammatory conditions has not been successful thus far. However, staging of malignancies by MRI has been as accurate as CT in this area. At this time, transrectal ultrasonography is the imaging modality of choice to screen for prostate gland carcinoma.

In the female pelvis, the uterus and ovaries are evaluated best by ultrasonography. MRI does demonstrate these structures well, however, and it may 
be used to stage or to further delineate masses. The signal characteristics of the endometrium have been shown to change with hormonal therapy and the menstrual cycle. ${ }^{26,27}$

Although MRI has not been demonstrated to have any biologic effect at the levels of magnetization or signal power used in imaging, a study of pregnant women should not be performed unless certain specific diseases, such as abdominal masses or pregnancy-related diseases, are being evaluated. Masses in the region of the gravid uterus are well demonstrated and may be characterized by MRI.

\section{Summary}

MRI is highly useful for visualizing the CNS; it swiftly is becoming a useful imaging modality outside of it also. Almost daily, discoveries are being made to overcome the shortfalls of image degradation from motion. The ability to image using cardiac gating provides a new, noninvasive way to evaluate the cardiovascular system.

MRI presently is going through a stage similar imaging from CNS imaging. The many diagnostic capabilities of MRI are just beginning to be known. In the new future, it may be possible to perform noninvasive, in vivo, tissue-specific diagnosis. noninvasive, in vivo, tissue-specific diagnosis.

1. Ramsey RG, Zacharias CE: MR imaging of the spine after radiation therapy: Easily recognizable effects. AJR 1985;144:1131-1135.

2. Mitchell DG, Rao VM, Dalinka M, et al: Hematopoietic and fatty bone marrow distribution in the normal and ischemic hip: New observations with 1.5-T MR imaging. Radiology 1986;161:199-202.

3. Mitchell DG, Kundel HL, Steinberg ME, et al: Avascular necrosis of the hip: Comparison of MR, CT, and scintigraphy. AJR 1986;147:67-71. 4. Mitchell DG, Kressel HY, Arger PH, et al: Avascular necrosis of the femoral head: Morphologic assessment by MR imaging, with CT correlation. Radiology 1986;161:739-742.

5. Rao VM, Fishman M, Mitchell DG, et al: Painful sickle cell crisis: Bone marrow patterns observed with MR imaging. Radiology 1986;161:211-215.

6. Reicher MA, Bassett LW, Gold RH, et al: High-resolution magnetic resonance imaging of the knee joint. Pathological correlations. AJR 1985;145:903-909.

7. Beltran J, Noto AM, Mosure JC, et al: The knee: Surface-coil MR imaging at 1.5 T. Radiology 1986;159:747-751.

8. Katzberg RW, Bessette RW, Tallents RH, et al: Normal and abnormal temporomandibular joint: MR imaging with surface coils. Radiology 1986;158:183-189.

9. Harms SE, Wilk RM, Wolford LM, et al: The temporomandibular joint: Magnetic resonance imaging using surface coil. Radiology 1985;157:133136.

10. Seeger LL, Ruszkowski JT, Bassett LW, et al: MR imaging of the normal shoulder: Anatomic correlation. AJR 1987;148:83-91.

11. Binkovitz LA, Ehman RL, Cahill DR, et al: Magnetic resonance imaging of the wrist. Radiographics 1988;8:1171-1202.

12. Beltran J, Noto AM, Mosure JC, et al: Ankle: Surface coil MR imaging at 1.5 T. Radiology 1986;161:203-209.

13. Atlan GH, Sigal R, Hadar H, et al: Nuclear magnetic resonance proton imaging of bone pathology. J Nucl Med 1986;27:207-215.

14. Sechtem U, Pflugfelder PW, White RD, et al: Cine MR imaging: Potential for the evaluation of cardiovascular function. AJR 1987;148:239. 246.

15. Fisher MR, McNamara MT, Higgins CB: Acute myocardial infarction: MR evaluation in 29 patients. AJR 1987;148:247-251.
16. Julsrud PR, Ehman RL: The broken ring sign in magnetic resonance imaging of partial anomalous pulmonary venous connection to the superior vena cava. Mayo Clin Proc 1985;60:874-879.

17. Dumoulin CL, Souza SP, Feng H: Multi-echo magnetic resonance angiography. Magn Reson Med 1987;5:47-57.

18. Zerhouni EA, Fishman EK, Jones R, et al: MR imaging of sterilized lymphoma, abstract. Presented at Radiologic Society of North America scientific program, Chicago, November 30-December 5, 1986.

19. Stark DD, Ferrucci ST: Technical and clinical progress in MRI of the abdomen. Diagn Imaging 1985;7:118-127.

20. Haaga JR: Magnetic resonance imaging of the liver. Radiol Clin North Am 1984;22:879-890.

21. Stark DD, Moseley ME, Bacon BR et al: Magnetic resonance imaging and spectroscopy of hepatic iron overload. Radiology 1985;154:137142.

22. Hess CF, Griebel J, Kurtz B, et al: Spleen involvement by malignant lymphoma: Improved detection by MR imaging compared with ultrasound and dynamic-CT scanning, abstract. Presented at Radiologic Society of North America scientific program, Chicago, November 30December 5, 1986.

23. Glazer GM, Woolsey EJ, Borrello J, et al: Adrenal tissue characterization using MR imaging. Radiology 1986;158:73-79.

24. Reinig JW, Doppman JL, Dwyer AJ, et al: Adrenal masses differentiated by MR. Radiology 1986;158:81-84.

25. Lupetin AR: Magnetic resonance appearance of the kidneys in paroxysmal nocturnal hemoglobinuria. Urol Radiol 1986;8:101-103.

26. Lee JK, Gerseill DJ, Balfe DM, et al: The uterus: In vitro MRanatomic correlation of normal and abnormal specimens. Radiology 1985;157:175-179.

27. Hricak H, Demas BE, Braga CA, et al: Gestational trophoblastic neoplasm of the uterus: MR assessment. Radiology 1986;161:11-16.

From the Department of Radiology, Michigan State University College of Osteopathic Medicine, East Lansing, Mich.

Reprint requests to Dr Pera, B220 Clinical Center, MSUCOM, East Lansing, MI 48824. 


\section{EFFICACY...SAFETY...EXPERIENCE}

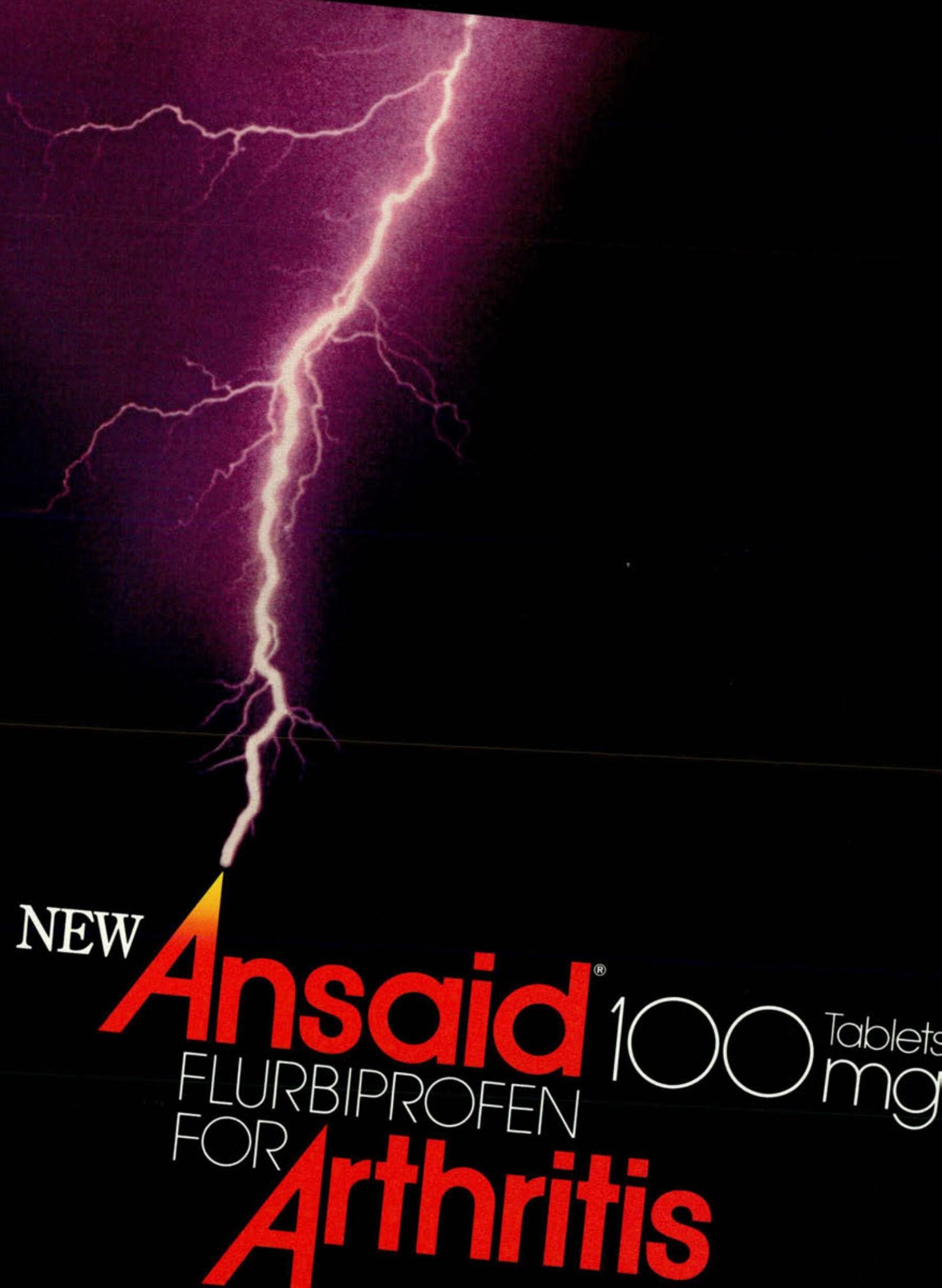




\section{Ansaid $100_{m g} 1$ Tablet BID or TID}

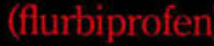

for Osteoarthritis

\section{Ansaid Tablets lessen disability in osteoarthritis}

Reduction of disability (investigators' assessment) in a 12-week, double-blind, randomized study of 147 patients with osteoarthritis of the knee treated with Ansaid, up to $200 \mathrm{mg} /$ day or aspirin, up to $4,000 \mathrm{mg} /$ day. $^{1}$

\section{Percent patients improved}

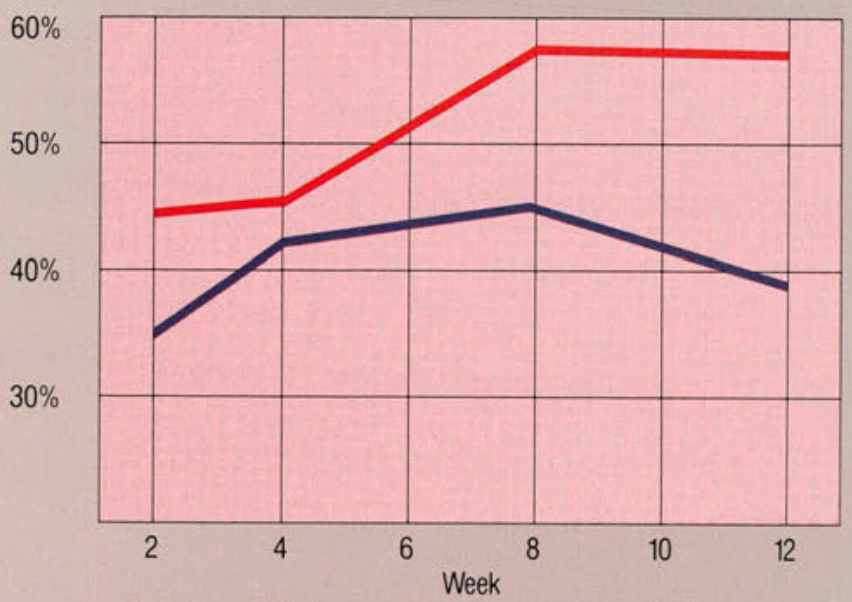

Ansaid $_{(n=63)}$
Ansaid (flurbiprofen) “... is an effective drug in controlling the pain and other symptoms of osteoarthritis of the knee."

\section{Convenient Dosage}

The dosage of Ansaid tablets is $200 \mathrm{mg}$ or $300 \mathrm{mg}$ daily, administered BID or TID. (Most experience in RA has been with TID or QID dosage.)

Recommended starting dose is $100 \mathrm{mg}$ BID.

\section{Efficacy maintained} week after week 


\section{EFFICACY, \\ SAFETY, \\ EXPERIENCE...}

\section{An excellent safety record worldwide}

- A safety profile derived from 4,123 patients who received flurbiprofen.

- 948 of these patients were treated for at least 6 months.

- Overall incidence of side effects with

Ansaid is about half that reported with comparable dosages of aspirin.

- Does not accumulate...excretion virtually complete 24 hours after the last dose.

Incidence of side effects in three body systems during clinical trials with Ansaid
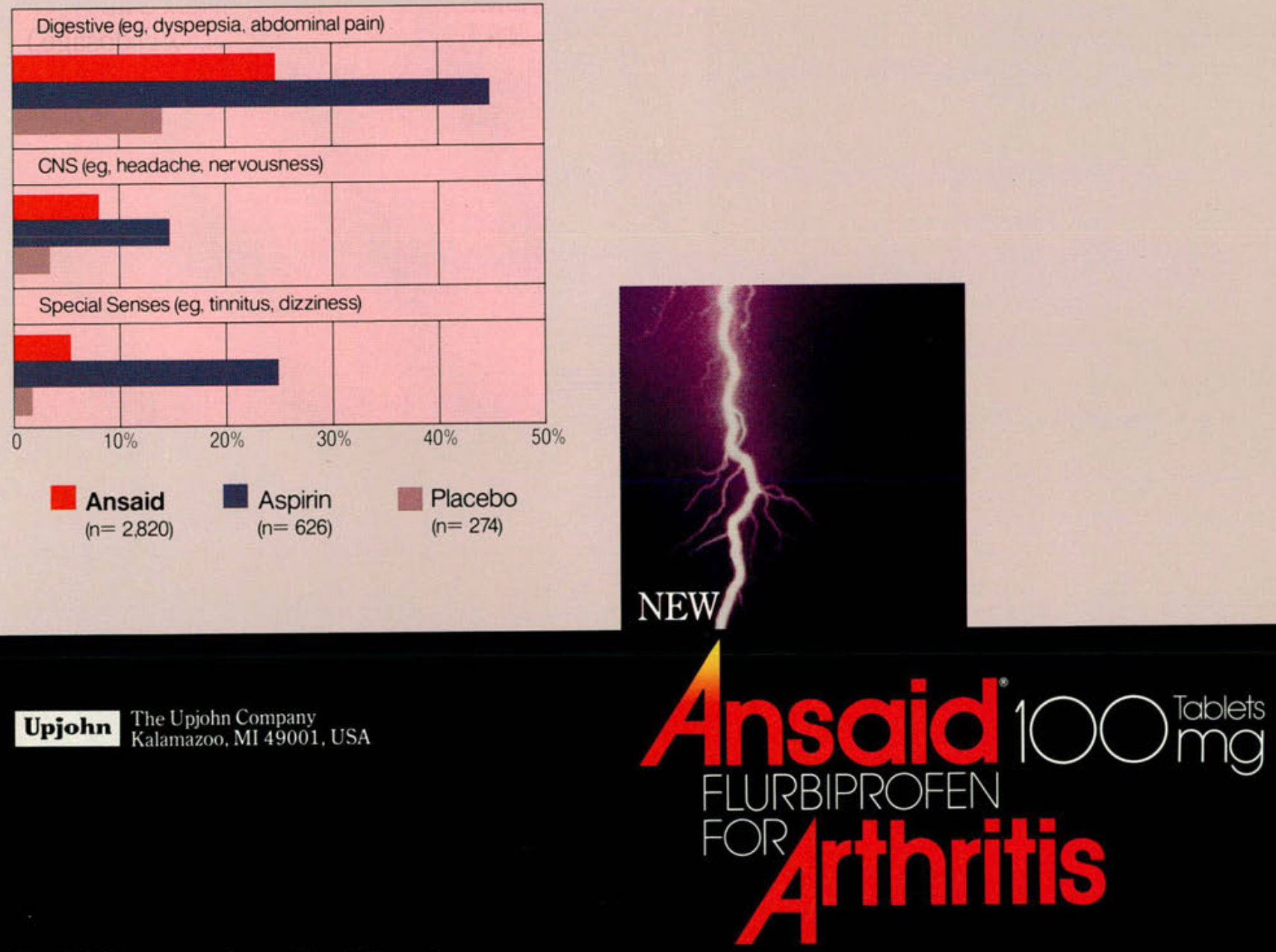


\section{Ansaid $100_{\text {mg: }} 1$ Tablet BID or TID}

\section{for Rheumatoid Arthritis}

Ansaid Tablets: Effective in acute and chronic phases

Results of a 52-week, double-blind, randomized study of 822 patients in the United States, demonstrated that Ansaid 200 to $300 \mathrm{mg} /$ day relieves rheumatoid arthritis pain and inflammation as effectively as comparable dosages of aspirin.

\section{Convenient Dosage}

- The dosage of Ansaid tablets is $200 \mathrm{mg}$ or $300 \mathrm{mg}$ daily, administered BID or TID. (Most experience in RA has been with TID or QID dosage.)

Recommended starting dose is $100 \mathrm{mg}$ BID.

- Results of a 12-week study of 143 patients with rheumatoid arthritis comparing BID and QID dosing of Ansaid (flurbiprofen) $200 \mathrm{mg}$ daily indicated the BID dosing of Ansaid is as effective as QID dosing?

\section{Improvement in efficacy parameters}

Mean percent change from baseline

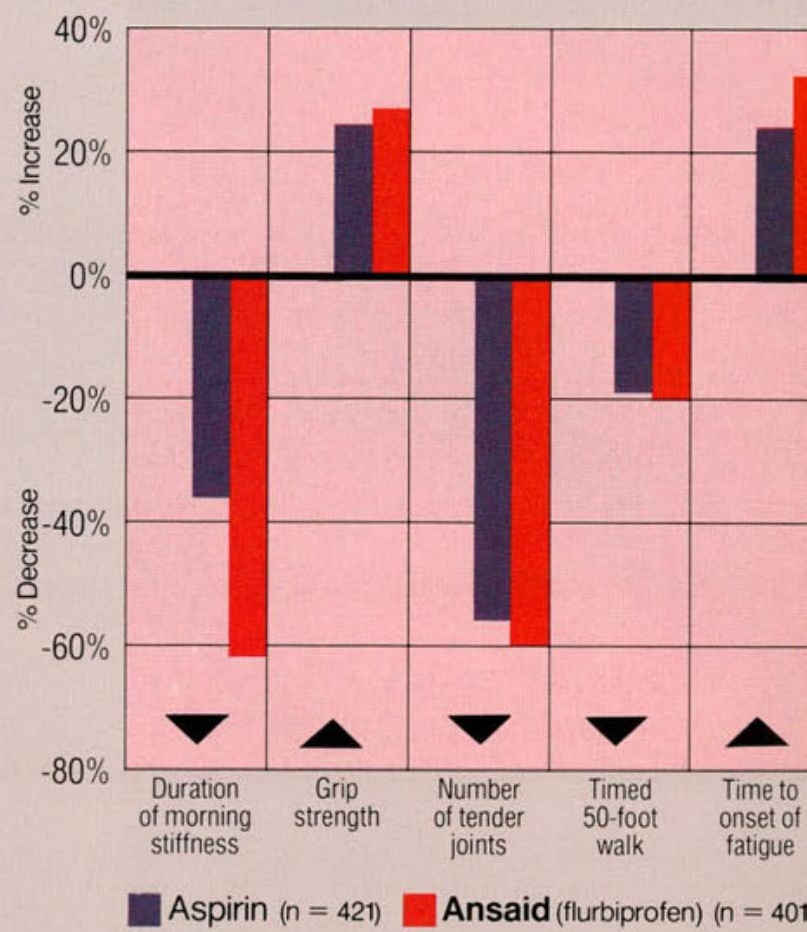

\section{Effective...improves patient mobility.}




\section{EFFICACY, \\ SAFETY, \\ EXPERIENCE...}

\section{Over a decade of worldwide experience}

- Experience in 70 countries

- For over a decade, flurbiprofen has been a widely used antiarthritic agent.

- Since 1977, there have been more than 1 billion patient-days of treatment with flurbiprofen throughout the world.

- An 8-year study involving 1,396 patients in the United Kingdom confirms the efficacy and safety of flurbiprofen in arthritis. $^{3-5}$

- A multicenter study of 11,500 arthritic patients in France provides additional confirmation of flurbiprofen efficacy and safety. ${ }^{6}$

- In the United States, controlled trials involving 2,820 patients further demonstrated the clinical efficacy and safety of Ansaid (flurbiprofen).
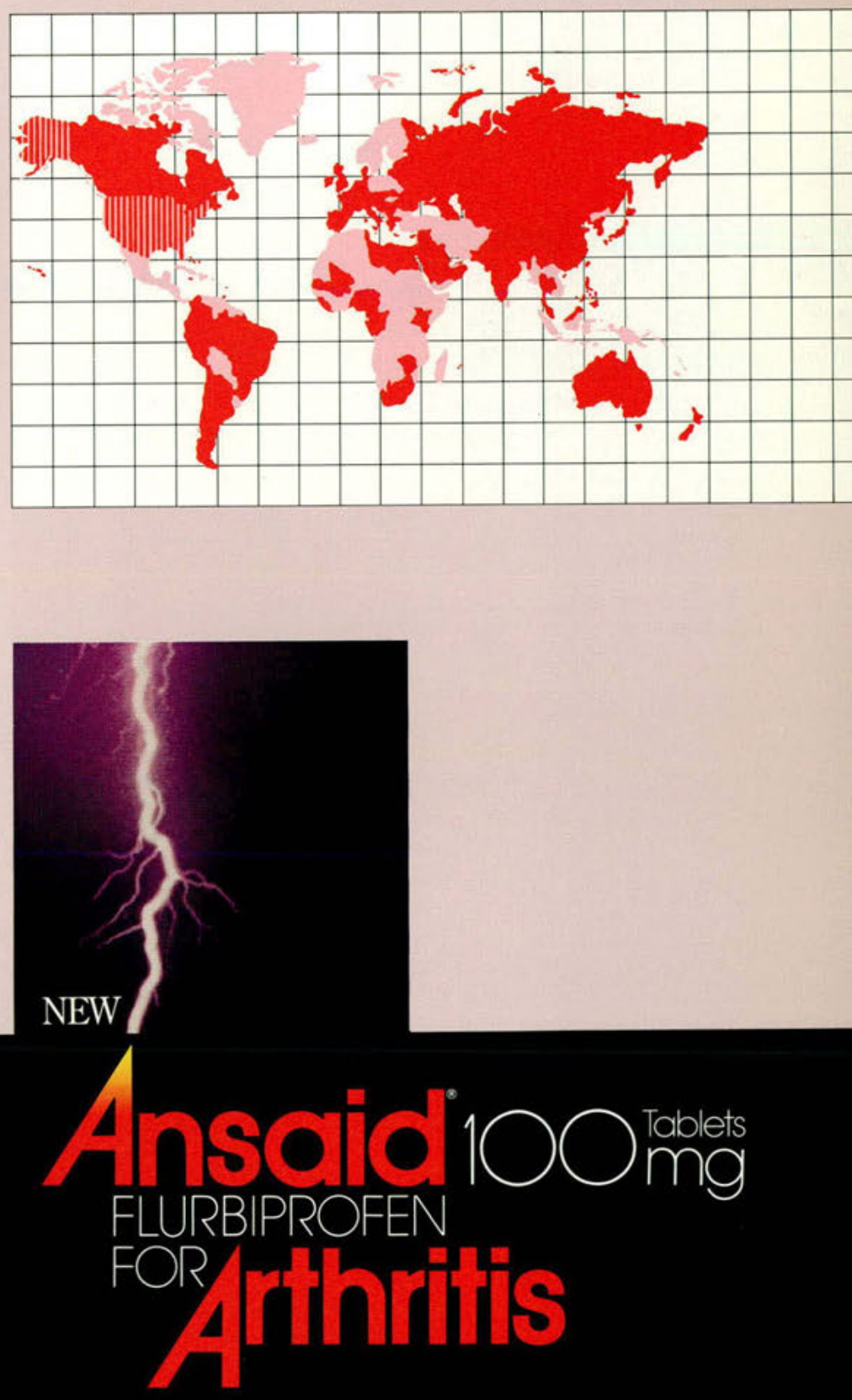


\section{EFFICACY, SAFETY, EXPERIENCE...}

The dosage of Ansaid tablets is $200 \mathrm{mg}$ or $300 \mathrm{mg}$ daily, administered BID or TID. (Most experience in RA has been with TID or QID dosage.)

Recommended starting dose is $100 \mathrm{mg}$ BID.

\section{ANSAID® Tablets}

(flurbiprofen)

INDICATIONS: Acute and long term treatment of signs and symptoms of rheumatoid arthritis and osteoarthritis. CONTRAINDICATIONS: Hypersensitivity to ANSAID, or if aspirin or any other nonsteroidal anti-inflammatory agent induces asthma, urticaria or other allergic type reactions Fatal asthmatic reactions have been reported in such patients. WARNINGS: Gastrointestinal effects: Risk of Gl ulcerations, bleeding and perforation with nonsteroidal anti-inflammatory therapy: Serious GI toxicity can occur at any time, with or without warning symptoms, during chronic treatment. The occurrence is about $1 \%$ after $3-6$ months, $2-4 \%$ after a year. Patients should be informed of signs and symptoms of serious Gl toxicity and what to do if it occurs. No subset of patients not at risk has been identified. Prior history of serious Gl events and other risk factors of peptic ulcer disease, e.g., alcoholism, smoking, etc., have been associated with increased risk. The elderly and debilitated tolerate ulceration and bleeding less well. Higher doses probably carry a greater risk. Gl ulceration and bleeding can occur without warning symptoms and chronically treated patients should be followed. PRECAUTIONS: Patients with impaired renal or hepatic function: Use ANSAID and similar agents cautiously Pharmacokinetics have not been studied in patients with decreased liver function. Renal Effects: Rats develop renal papillary necrosis at dosages equivalent to human therapeutic levels, as do monkeys given 20-40 times the human dose. In clinical studies of ANSAID, kidney function tests were done monthly and renal effects were similar to those seen with other nonsteroidal anti-inflammatory drugs. A second form of renal toxicity has been seen in patients with prerenal conditions that reduce renal blood flow or blood volume. A nonsteroidal anti-inflammatory drug may cause dose-dependent reduction in prostaglandin formation and precipitate overt renal decompensation. Patients at greatest risk are those with impaired renal or hepatic function, heart failure, those taking diuretics or the elderly. Drug discontinuation usually leads to recovery. Patients at high risk on chronic treatment should have renal function monitored if they have signs or symptoms that may be consistent with mild azotemia, e.g., malaise, fatigue, loss of appetite. Occasionally BUN and serum creatinine may be elevated without signs or symptoms. Flurbiprofen is excreted by the kidneys and pharmacokinetics are changed by renal failure so patients with renal failure should be monitored and may require a reduction of dosage to avoid accumulation of flurbiprofen metabolites. Liver tests: Borderline elevations of liver function tests may occur in up to $15 \%$ o patients, and may progress, remain unchanged or disappear with continued treatment. Patients with signs and/or symptoms or with an abnormal liver function test should be evaluated further Anemia: Patients treated long term who have initial hemoglobin values under $10 \mathrm{~g} / \mathrm{dL}$, should have periodic hemoglobin values. Fluid retention and edema: Fluid retention and edema have been reported so use ANSAID with caution in patients with conditions such as cardiac decompensation or hypertension. Vision Changes: Blurred and/or diminished vision has been reported. Patients with eye complaints should have periodic ophthalmologic exams. Effect on platelets and coagulation: Platelet aggregation is inhibited and bleeding time prolonged patients who may be adversely affected should be carefully observed. Information for patients: Physicians and patients may wish to discuss potential risks and likely benefits. Drug Interactions: Anticoagulants: Bleeding parameters are affected, clinical bleeding has been reported. Aspirin: Flurbiprofen levels were $50 \%$ lower. Concurrent use is not recommended. Betaadrenergic Blockers: Pharmacokinetics and heart rate reduction are not affected, hypotensive effect of propranolol but not atenolol was attenuated. Cimetidine, Ranitidine: Cimetidine causes a 13\% increase in area under the flurbiprofen serum concentration curve. Diuretics: Patients receiving furosemide or thiazides should be closely observed to make sure the desired effect is obtained.

\section{References}

1. Lomen PL, Lamborn KR, Porter GH, et al: Treatment of osteoarthritis of the knee: A comparison of flurbi-

profen and aspirin. $A m J$ Med 1986; 80(suppl 3A): 97-102. 2. Brown BL, Daenzer CL, Hearron MS, et al: Comparison of two dosing schedules of flurbiprofen for patients with rheumatoid arthritis. Am J Med 1986; 80(suppl 3A): 19-22. 3. Busson M: A long-term study of flurbiprofen in rheumatological disorders: I. Rheumatoid arthritis. JInt Med Res 1986;14:1-6.

4. Busson M: A long-term study of flurbiprofen in rheumatological disorders: II. Osteoarthritis. IInt Med Res 1986;14:7-12. 5. Busson M: A long-term study of flurbiprofen in rheumatological disorders: III. Other articular conditions.

Jint Med Res 1986;14:13-18.

6. Kieffer G: Flurbiprofen: A multicenter trial in 11,500 ambulatory patients (French). Rev Med 1981:22:915-924.
Carcinogenesis, mutagenesis, impairment of fertility: No evidence. Teratogenic effects: Pregnancy category B: No effect in animals. Not recommended for use in pregnancy Labor and delivery, nursing mothers, pediatric use: Use is not recommended. ADVERSE REACTIONS: $9.4 \%$ of 4123 patients dropped out of studies because of an a.d.r. Incidence $>1 \%$ : Gastrointestinal: Dyspepsia*, diarrhea*, abdominal pain* nausea* constipation bleeding, flatulence, elevated liver enzymes and vomiting. Central nervous system: Headache* "stimulation" (e.g., anxiety, insomnia, reflexes increased, tremor) and "inhibition" (e.g., amnesia, asthenia, somnolence, malaise and depression). Respiratory: Rhinitis. Dermatologic: Rash. Special senses: Dizziness, tinnitus and changes in vision. Genitourinary: Signs and symptoms suggesting a urinary tract infection* Body as a whole: Edema* Metabolic/nutritional: Body weight changes. *Reaction in 3 to $7 \%$ of patients. Incidence $<1 \%$ (Causal relationship probable): Gastrointestinal: Peptic ulcer disease (See Warnings), gastritis, bloody diarrhea stomatitis, esophageal disease, hematemesis and hepatitis, cholestatic and non-cholestatic jaundice. Central nervous system: Ataxia, cerebrovascular ischemia, confusion, paresthesia and twitching. Hematologic: Decrease in hemoglobin and hematocrit, iron eosinophilia and ecchymosis, thrombocytopenia, hemolytic anemia and aplastic anemia. (See Precautions) Respiratory: leukopenia, anemia, deficiency Asthma and epistaxis. Dermatologic: Angioedema, urticaria, eczema and pruritus; photosensitivity, toxic epidermal necrolysis and exfoliative dermatitis. Special senses: Conjunctivitis and parosmia. Genitourinary: Hematuria and impairment of renal function, interstitial nephritis. Body as a whole: Anaphylactic reactions, chills, fever. Metabolic Nutritional: Hyperuricemia. Cardiovascular: Heart failure, hypertension, vascular disease and vasodilatation. Incidence <1\% (Causal relationship unknown): Gastrointestinal: Periodontal abscess, appetite changes, cholecystitis and dry mouth. CNS: Convulsion, meningitis, hypertonia, cerebrovascular accident, emotional lability and subarachnoid hemorrhage. Hematologic: Lymphadenopathy. Respiratory: Bronchitis, laryngitis, dyspnea, pulmonary embolism, pulmonary infarct, hyperventilation. Dermatologic: Alopecia, nail disorder, herpes, dry skin and sweating. Special senses: Ear disease, corneal opacity, glaucoma, retrobulbar neuritis, change in taste, transient hearing loss, retinal hemorrhage. Genitourinary: Menstrual disturbances, vaginal and uterine hemorrhage. vulvovaginitis, prostate disease. Metabolic nutritional: Hyperkalemia Cardiovascular: Arrhythmias, angina pectoris and myocardial infarction. Musculoskeletal: Myasthenia. DOSAGE AND ADMINISTRATION: 200 to $300 \mathrm{mg}$ daily, administered bid, tid or qid. (Most experience in rheumatoid arthritis has been with tid or qid dosage). Dose should be tailored to severity of symptoms and patient response. Store at controlled room temperature $\left(15-30^{\circ} \mathrm{C}\right)$. Federal law prohibits dispensing without a prescription.

\section{Upjohn}

The Upjohn Company

Kalamazoo, MI 49001, USA

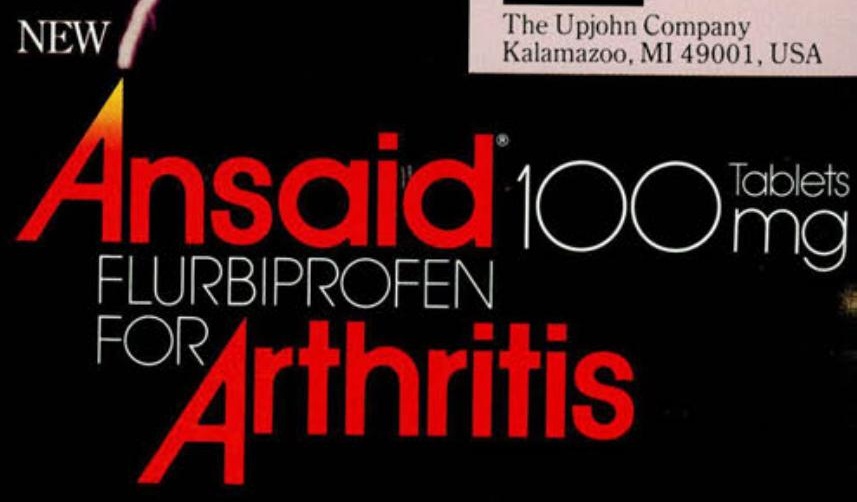

